\title{
Capturing complex vaccine-immune-disease relationships for free-ranging koalas: IL17 production is an important intermediate factor against chlamydial disease
}

David Lizárraga ( $\square$ David.Lizarraga@utas.edu.au )

University of Tasmania https://orcid.org/0000-0003-1158-9407

\section{Peter Timms}

University of the Sunshine Coast

\section{Bonnie L Quigley}

University of the Sunshine Coast

Jon Hanger

Endeavour Veterinary Ecology Pty Ltd

\section{Scott Carver}

University of Tasmania

\section{Research}

Keywords: structural equation model, Chlamydia, vaccine, koala, cytokine

Posted Date: January 20th, 2020

DOl: https://doi.org/10.21203/rs.2.21243/v1

License: (c) (1) This work is licensed under a Creative Commons Attribution 4.0 International License.

Read Full License 


\section{Abstract}

Background Disease is a major factor negatively affecting koala populations. Vaccination is a promising treatment option that would result in immune-mediated protection against disease. Measuring and assessing vaccine efficacy can be challenging owing to both direct and indirect interactions caused by vaccination. In this study, we investigate vaccine-immune-chlamydial load-disease relationships from MOMP (major outer membrane protein) vaccine trials to protect healthy free-ranging koalas against Chlamydia -related diseases.

Methods We created a priori hypotheses based on data sources and perceived direct and indirect interactions from koalas vaccinated six months prior. Each hypothesis was tested as a structural equation model to evaluate possible causality among measured variables. Model averaging was used as multiple models fit the data, and the strength of relationships were examined through averaged coefficients and the raw data.

Results Despite multiple potential sources of variation owing to the koalas in this study being freeranging, our analyses provide unique insights into the effects of vaccinating against Chlamydia. We found more relationships in urogenital models with interleukin 17 (IL17) production compared to models with interferon gamma (IFNY) production. In the averaged model with IL17, urogenital chlamydial load was positively associated with disease and negatively associated with IL17 production. MOMP vaccination had a trending effect for reducing chlamydial load and also had a strong effect on increasing IL17 production. This indicated a link between MOMP vaccination, reduction of urogenital chlamydial load, and increased production of IL17. Not surprisingly, urogenital chlamydial load was a positive predictor for the development of urogenital disease at six months post-vaccination.

Conclusions Using structural equation modelling, this study has helped illuminate that the immune cytokine IL17 may be an important intermediate factor linking MOMP vaccination with chlamydial pathogenesis and protection, enhancing previous investigations. Going beyond univariate statistics, the methods used in this study can be applied to other preclinical vaccination experiments to identify important direct and indirect factors underpinning the effects of a vaccine.

\section{Introduction}

Koala (Phascolarctos cinereus) populations, particularly in areas of the Australian Capital Territory, New South Wales, and Queensland, have suffered staggering losses over recent years $[1,2,3]$ leading to their conservation status being listed as 'vulnerable' per the International Union for Conservation of Nature [4]. A number of factors negatively affect koala populations, including habitat loss, climate change, bushfires, motor vehicle accidents, dog attacks, and disease [5]. Deterministic age structured matrix models [6] of four of these factors (habitat loss, dog attacks, motor vehicle collisions, and disease) indicate that reducing the prevalence of disease may stabilize koala populations. The magnitude of disease-related mortality within a given population is potentially exacerbated by environmental stressors 
including climate change, habitat loss resulting from urbanisation, and environmental disasters such as bushfires, though to our knowledge no study has investigated this in wild koala populations [7]. Amid its variable magnitude, the reduction of disease has been a key management strategy of this species $[2,5,6$, 8].

Chlamydia is an obligate, intracellular bacterium that is the most prevalent disease-causing pathogen in wild koalas $[5,9]$. Koalas are hosts to two bacterial species of Chlamydia (C. pecorum and $C$. pneumoniae), however, modern vaccine candidates target the more prevalent species, $C$. pecorum, that typically infects epithelial cells in the ocular and urogenital mucosa [10]. Sterility and disease-related mortality as a result of chlamydial infections have a direct, negative impact on koala population dynamics and are relatively common among some free-ranging koala populations $[8,11]$. Chlamydial infections in koalas are treatable with antibiotics, but this treatment strategy is potentially fatal for the specialized microflora in the koala gastrointestinal tract that is necessary for eucalyptus digestion [12]. Vaccinating koalas is a promising treatment strategy that is modelled to have clear benefits, particularly at the population scale. Modelling by Craig et al. [13] suggested that chlamydial vaccination could stabilize koala populations after five years of a vaccine program (with $75 \%$ efficiency) administered to around $10 \%$ of koalas per year. Such a vaccine does not yet exist, however, many studies within the last decade have advanced the development of a vaccine for koalas against Chlamydia.

Much of the foundational work for chlamydial vaccine development has used captive koalas under controlled veterinary conditions (reviewed by Phillips et al. [14]). However, field trials that encompass a greater range of natural variables provide a more accurate picture of vaccine efficacy. To date, there are two published field trials testing a chlamydial vaccine using free-ranging koalas. The first study was by Waugh et al. [15]. This vaccine consisted of three chlamydial major outer membrane proteins (MOMP) from three genotypes (A, F, and G) and an Immunostimulating Complex (ISC) adjuvant, that was delivered subcutaneously three times over three months (given at one-month intervals). Chlamydial load and disease were compared six months post-vaccination between vaccinated and control koalas in the 60 koala experiment. Vaccinated animals had increases in anti-Chlamydia immunoglobulin G (IgG) and lower levels of chlamydial load and prevalence of disease (at both the ocular and urogenital sites) compared to control koalas six months after the initial vaccination. The second field study was by Desclozeaux et al. [16]. This trial tested two vaccine formulations consisting of either MOMP (three genotypes A, F, and G) or PMP (peripheral membrane protein, genotype $G$ ). Both vaccines were delivered subcutaneously alongside a tri-adjuvant (PCEP, IDR1002, and polyl:C) and vaccinated groups were compared to non-vaccinated control koalas (21 koalas in each group, 63 in total). Both MOMP and PMP vaccinated koalas had elevated interferon gamma (IFNY) and interleukin (IL) 17 production six months post-vaccination. Additionally, koalas vaccinated with MOMP had lower chlamydial loads compared to control koalas six months post-vaccination. Combined, these field trials have examined enough koalas that, for the first time, more detailed modelling analysis of factors involved in vaccine responses in koalas can be considered. 
The koala immune response has been a focal point of research in recent years, particularly to chlamydial infection (reviewed by Madden et al. [17]). Several important cytokines or antibodies in response to chlamydial infection in koalas have been identified: IFNy, IL17, IL10, tumor necrosis factor alpha (TNFa), $\operatorname{lgG}$, and IgA. As multiple aspects of the koala immune response are poorly understood, researchers often refer to vaccine trials against two other chlamydial species, $C$. trachomatis or $C$. muridarum, where mice are most often used as host models of infection $[18,19]$. Of these murine trials, the most commonly measured host cytokine in response to chlamydial infection is IFNY [18], where the production of IFNY has been associated with protection against chlamydial disease [20]. Increases in IFNy concentration in vitro can lead to the degradation of tryptophan, leading to the starvation of $C$. trachomatis of this essential amino acid and inducing chlamydial persistence (an inactive, intracellular pathogen response to external stressors; [21]). A recent study has shown $C$. pecorum to be resistant to increasing concentrations of IFNY in vitro, suggestive of different immune-evasion mechanisms as compared to $C$. trachomatis [22]. This difference from $C$. trachomatis (which is sensitive to IFNY) could lead to a different effect of IFNY in koalas to $C$. pecorum infection. Lastly, in murine hosts, elevated levels of both IFNY and IL17 increased the production of inducible Nitric Oxide synthase (iNOS), promoting the production of microbicidal nitric oxide (NO) that correlated with the reduction of chlamydial load [23]. Some studies, however, have suggested that an elevated iNOS response to infection (elevated by host cytokines) may be associated with scarring of the fallopian tubes and immunopathogenesis [24, 25]. The relationships between chlamydial vaccination, the host immune response (particularly IFNY and IL17), chlamydial load, and disease is still poorly understood in koalas.

Clearly, a key challenge in chlamydial vaccine research is understanding complex direct and indirect immune-mediated control of infection and disease. In this study, we aimed to model important direct and indirect factors surrounding the vaccination of free-ranging koalas: host immune parameter, chlamydial load, and disease. We used structural equation models to identify the directionality and magnitude of direct and indirect relationships when all four variables are modelled together. This statistical method has been used previously to identify both multiple environmental and individual factors that affect chlamydial disease pathology in koalas [26]. We modelled vaccination status, the production of an immune parameter (either IFNy or IL17), chlamydial load, and disease status at two important mucosal sites (ocular and urogenital) using data collected by Waugh et al. [15] and Desclozeaux et al. [16]. We tested six hypotheses to 1) identify the relationships between immune parameter and chlamydial load, and 2) identify the relationships between host immune parameter and disease.

\section{Methods}

\section{Pooling data from both field trials}

Previously collected data from two field trials of free-ranging koalas vaccinated against Chlamydia described by Waugh et al. [15] and Desclozeaux et al. [16] were used in our analysis (see Appendix 1 for a 
summary of the methods for both studies). The two field trials (different vaccination schedules and different adjuvants) were investigated to determine if differences exist in the data for MOMP vaccinated individuals (PMP vaccinated individuals were excluded) using both a meta-analysis and a univariate approach (Figure 1). An effect size (or a Hedge's $g$ ) and variance were calculated to estimate the effect of MOMP vaccination on chlamydial load and disease at both the ocular and urogenital sites for both field trials (a total of 8 effect sizes; see Borenstein et al. [27]). A meta-regression was performed on these effect sizes using the MAd package [28] in the statistical program R (v3.5.3; [29]). As control koalas in the field trial by Desclozeaux et al. [16] did not have measurements of IFNy or IL17 production, a comparison was made for these immune measurements using univariate methods. As no evidence indicated differences between the two trials, the data collected from Waugh et al. [15] and Desclozeaux et al. [16] were pooled to test hypothesized models with chlamydial load, disease, and immune response measurements taken at six months after the first chlamydial vaccination.

\section{Creation and testing of hypothesized models}

Six a priori structural equation models were created based on biological hypotheses proposed in the literature (Table 1). To begin, IFNy and IL17 production were evaluated as the immune parameters. Two models were made to determine if, 1) a causal relationship exists between these immune parameters and disease, and 2) the directionality between these immune parameters and chlamydial load (hypotheses 1 and 2). Four additional models were made to assess the directionality between 1) chlamydial load and these immune parameters, and 2) these immune parameters and disease (hypotheses $3,4,5$, and 6 ). Only one immune parameter was included at a time in each of the six hypothesized models so as to maintain appropriate 10:1 sample size to variable ratios [30], resulting in 30 different models. The data were separated by either the urogenital or ocular site and tested in each hypothesized model, which resulted in a total of 60 models tested. The ocular models include ocular chlamydial load and disease status (e.g. ocular conjunctivitis), and the urogenital models include urogenital chlamydial load and disease status (e.g. cystitis or dilation of the ovarian bursae).

Prior to model testing, we chose to standardize data from all variables into a 0 to 1 scale to make all model coefficients between all variables comparable within each model. Binomial values were assigned to vaccination status ( 0 for non-vaccinated and 1 for vaccinated), and ocular and urogenital disease status ( 0 for clinical disease absent or subclinical and 1 for clinical disease present). Ocular and urogenital chlamydial load measurements were placed in one of three ordinal categories from 0 to 1 depending on an untransformed qPCR result similar to those used by Quigley et al. [26]: 1) samples with no detectable qPCR result were given a value of 0,2 ) samples with $\leq 100$ copies $\mu \mathrm{L}^{-1}$ were detectable but not quantifiable and given a value of 0.5 , and 3 ) samples with $>100$ copies $\mu \mathrm{L}^{-1}$ were detectable and quantifiable and given a value of 1 . Finally, values for both IFNy and IL17 production were transformed to a 0 to 1 scale by dividing each measurement by the maximum IFNy or IL17 measurement, respectively. 
Structural equation modelling (SEM) requires an individual to have all measurements for each variable tested in the model. We first tested each of the 60 models for model fit using the fit indices Root Mean Square Error of Approximation (RMSEA, absolute fit index) and Confirmatory Fit Index (CFI, relative fit index) described by Kline [31], and a Bollen-Stine Bootstrap for non-parametric model fit (100 bootstrap draws; [32, 33]). No further analysis was performed for models that failed to fit the data using these indices (CFI > 0.9; RMSEA < 0.05; Bollen-Stine p-value > 0.05; [31, 32]). Models that fit the data, however, were then compared using Akaike's information criterion corrected for small sample sizes (AICC) and model weights $\left(\mathrm{w}_{\mathrm{i}}\right)$ were calculated $[34,35]$. All models were tested using R statistical software (v3.5.3; [29]) using the lavaan package [36].

\section{Model interpretation}

A coefficient of determination $\left(R^{2}\right)$ was obtained for disease, chlamydial load, and immune parameter production for each of the fitting models to estimate how well the model predicts each of these variables within the model. All standardized relationship coefficients $( \pm 95 \% \mathrm{Cl})$ were obtained for each path tested in each of the fitting models. Standardized relationship coefficients indicate the strength of the relationship (the magnitude of the coefficient), direction of the relationship (e.g. positive relationships indicate positive effects of one variable on another), and how well each relationship is predicted (by the $95 \% \mathrm{Cl}$ ) by the model. We used standardized relationships in our model interpretation so we could compare the magnitude of all relationships within each model. As some models contained different sample sizes, care was taken not to compare relationship coefficients (or $\mathrm{R}^{2}$ ) across unrelatable models (i.e. models that do not contain the same variables). We defined strong model relationships based on $95 \% \mathrm{Cl}$ and whether it crossed 0 , the threshold of an effect of an exogenous (predictor) variable on an endogenous variable (response). As our models were similar in complexity and limited by sample size, it was not uncommon that more than one model fit the data. For multiple model interpretation, we used model averaging to calculate model averaged parameters (and variance, 95\% $\mathrm{Cl}$ ) using the previously described model weights (see Burnham et al. [35] for equation).

\section{Results}

We first investigated the effects of MOMP vaccination (six months after the initial vaccination) on chlamydial abundance and disease at two important mucosal sites (ocular and urogenital) between two chlamydial vaccination trials using free-ranging koalas $[15,16]$. We estimated effect sizes (as Hedge's g) for these responses (see Methods; [18]) and found no evidence for a difference in MOMP vaccination on chlamydial load and disease between field trials (meta-regression, $p=0.956$; Figure 1a). Additionally, we found measurements of IFNy or IL17 production to be comparable between the two experiments after performing a non-parametric Wilcoxon rank sum test (IFNy, $p=0.9095 ; \mathrm{IL} 17, p=0.1191$; Figure 1b). As we found no evidence supporting a difference between the two trials, we pooled the data to obtain sample sizes sufficient to use in structural equation models. 
The pooled data from the two trials resulted in 91 MOMP vaccinated or control wild koalas with recorded clinical disease status and measured chlamydial load out to six months post-vaccination. Of these 91 koalas, a subset of animals had measurements of either IFNY $(n=40)$ or IL17 $(n=36)$ production. We focussed on models with both immune parameters analysed separately as structural equation modelling requires robust sample sizes (i.e. ratio of samples to variables analysed $=10: 1$, [30]; Appendix 2). When we tested the 6 hypotheses for both the ocular and urogenital sites with either IFNy or IL17 production (Table 1), we obtained multiple models that fit the data (Appendix 3). For multiple hypothesis interpretation, we focussed on coefficients where the $95 \%$ confidence interval did not overlap 0 (a strategy used in other structural equation modelling studies such as Dorresteijn et al. [37]). Additionally, we used a model averaging approach to determine if the direction and magnitude of these relationships were consistent across multiple models.

Models with ocular chlamydial load and disease: MOMP vaccination reduced ocular chlamydial load

All six models with ocular chlamydial load, disease, and IFNy production fit the data (Appendix 3 ). We found that MOMP vaccination had a negative effect on chlamydial load in koalas (hypothesis 1, $-0.286 \pm$ 0.276 ; hyp. $2,-0.320 \pm 0.300$; hyp. $3,-0.320 \pm 0.288$; hyp. 6, $-0.320 \pm 0.259$; Table 2 ). We obtained the same result when all models were averaged together (model average, $-0.303 \pm 0.300$; Table 2 and Figure $2 a$ ).

Four models containing ocular chlamydial load, disease, and IL17 production fit the data (hypotheses 3 , 4, 5, and 6; Appendices 3, 4, and 5). Two of these hypotheses showed that koalas receiving a MOMP vaccination tended to have a lower chlamydial load (hypothesis $3,-0.320 \pm 0.304$; hyp. 6, $-0.320 \pm 0.314$; Table 2 and Figure 2c), agreeing with models containing IFNy production. Three hypotheses indicate that MOMP vaccinated koalas produced more IL17 compared to control koalas (hypothesis 3, $0.106 \pm 0.065$; hyp. 5, $0.166 \pm 0.104$; hyp. $6,0.130+0.100$ ), and koalas (either vaccinated or control) that had a high ocular chlamydial load tended to have low IL17 production $(-0.115 \pm 0.102$; hypothesis 6$)$. Lastly, one hypothesis supported a relationship between ocular disease and an increase in IL17 production (0.832 \pm 0.080 , hypothesis 3 ); this however, is complicated as only one koala developed ocular disease after 6 months. When all models were averaged, only one relationship had a marginal effect (ocular chlamydial load has an inverse relationship with IL17 production; -0.052 \pm 0.052 ; Table 2 and Figure $2 c$ ).

Models with urogenital chlamydial load and disease: MOMP vaccinated koalas reduced chlamydial load and had higher IL 17 production

Four models with urogenital chlamydial load, disease, and IFNy production that fit the data (hypotheses 3, 4, 5, and 6; Appendices 3, 5, and 6). When we investigated the coefficients for each relationship across all urogenital models with IFNy production, we found no clear relationships due to the large variance for each coefficient. As a result, we found no clear support for directionality or magnitude 
between IFNy production and urogenital chlamydial load or IFNy production and urogenital disease, though some relationships within these models were trending. We found a trending relationship between MOMP vaccinated koalas and a lower urogenital chlamydial load compared to control koalas $(-0.236 \pm$ 0.265 , Table 2 and Figure $2 b$ ).

There were four hypotheses with urogenital chlamydial load and disease and IL17 production that fit the data (hypotheses 3, 4, 5, and 6; Appendix 3). There was clear evidence that MOMP vaccinated koalas had a lower urogenital chlamydial load compared to control koalas (hypothesis $3,-0.320 \pm 0.261$ ) . MOMP vaccinated koalas produced more IL17 compared to control animals that was supported by all four hypotheses (hypothesis $3,0.112 \pm 0.084$; hyp. 4, $0.193 \pm 0.149$; hyp. 5, $0.166 \pm 0.110$; hyp. 6, $0.115 \pm$ 0.086 ; Table 2). These four hypotheses also indicate that more diseased koalas typically had a higher chlamydial load (hypothesis 3, $0.394 \pm 0.361$; hyp. 4, $0.563 \pm 0.537$; hyp. $5,0.541 \pm 0.386$; hyp. 6, $0.541 \pm$ 0.335 ; Table 2). Two hypotheses indicate that koalas (both control and vaccinated) with a high chlamydial load tended to produce less IL17 compared to koalas with a low chlamydial load (hypothesis $3,-0.244 \pm 0.178$; hyp. $6,-0.161 \pm 0.122$; Table 2). Lastly, across all hypotheses, we found relationship between IL17 production and urogenital disease. When all urogenital models with IL17 production were averaged we found: 1) that MOMP vaccinated koalas produced more IL17 compared to control koalas $(0.145 \pm 0.107), 2)$ koalas with a high chlamydial load were more likely to develop urogenital disease 6 months post-vaccination ( $0.506 \pm 0.407)$, and 3 ) koalas (both vaccinated and control) with a high urogenital chlamydial load tended to produce less IL17 compared to koalas with a lower urogenital chlamydial load $(-0.107 \pm 0.084$; Table 2 and Figure $2 \mathrm{~d})$. These structural equation models therefore connect relationships among MOMP vaccination, IL17 production, urogenital chlamydial load, and disease (Figure 2d).

\section{Discussion}

There exist many unknown aspects surrounding chlamydial vaccination in koalas primarily due to the difficulty in obtaining samples and the cost for vaccine experiments. Much of the underlying chlamydial biology, particularly complex immunological responses to infections, is understood from experiments using the mouse model or from in vitro cell cultures. In our current study, data from two free-ranging koala vaccination trials (both using the major outer membrane protein, MOMP) were used to investigate the causal relationships between 1) chlamydial infection and production of koala immune parameters, and 2) production of koala immune parameters and chlamydial disease. Despite sources of variation in this study (low numbers of koalas, use of outbred animals, unknown number of sexual encounters, unknown infection dates) there were clear relationships identified in our analyses that provide unique insights into the effects of vaccinating free-ranging koalas against Chlamydia. In particular, this study has helped establish the immune cytokine IL17 as an important factor connecting MOMP vaccination to chlamydial load and pathogenesis.

Two measurements of the koala immune response were incorporated into the models created in this study, IFNY and IL17 production. IFNy, a T-cell secreted cytokine, is currently regarded as one of most 
important cytokines in chlamydial biology [38]. Across all pre-clinical studies investigating a chlamydial vaccine using various animal models (mice, non-human primates, cats, pigs, guinea pigs etc.), there exists a common aim of eliciting strong mucosal T-cell responses, particularly increases to IFNY concentration $[14,18]$. Though IFNY is involved in multiple immune pathways, IFNY production leads to the enzymatic degradation of tryptophan, effectively starving Chlamydia of this essential amino acid [19]. Some studies suggest that tryptophan starvation may even cause $C$. trachomatis to enter a persistence form $[21,39]$. Experiments conducted in vitro show that elevated IFNy concentrations prevent chlamydial growth in $C$. trachomatis, but not $C$. pecorum [22]. Differing sensitivities to IFNy between these two chlamydial species might explain why measurements of koala IFNy production had no relationship with chlamydial load in any of our models (either ocular or urogenital; Table 2 and Figure 2a and 2b). This result is primarily due to the large amounts of variance that can be seen in the raw data (Appendix 5). The average log-transformed IFNy measurement between the non-vaccinated and vaccinated groups were similar, $1.378 \pm 0.918(95 \% \mathrm{Cl}, \mathrm{n}=16)$ and $1.445 \pm 0.430(95 \% \mathrm{Cl}, \mathrm{n}=25)$, respectively. Additionally, IFNy measurements have been shown to be dependent on the stage of chlamydial infection in mice [40] and non-human primates [41], something that is currently uncontrollable in trials using free-ranging koalas.

In contrast, models with IL17 production indicated that koalas that typically had lower ocular and urogenital chlamydial load tended to have higher levels of IL17 expression, six months post-vaccination (Table 2 and red arrows in Figure 2c and 2d). Experiments using IL17 knockout mice have shown that the production of IL17 is a factor in both protection [42] as well as pathogenesis [43]. IL17 is a proinflammatory cytokine secreted mainly from $\mathrm{T}$ cells and has many functions in response to chlamydial infection. In the wild type mouse model and in vitro experiments, IL17 has been shown to work synergistically with IFNy to inhibit $C$. muridarum growth by increasing the expression of inducible nitric oxide synthase (iNOS) and indirectly increasing antimicrobial nitric oxide (promoted by iNOS; [23]). Indeed, iNOS has been shown to be up-regulated in human patients suffering from ectopic pregnancies associated with $C$. trachomatis infection [24]. In koalas, one study reported the IL17a response from koala PBMCs to be higher in clinically diseased animals $(n=12)$ compared to subclinical animals $(n=29$; [44]). Three studies since have measured free-ranging koala IL17 response to chlamydial vaccination. In the first, Khan et al. [45] focussed on non-diseased koalas that tested negative for Chlamydia, finding that IL17 production increased with MOMP vaccination. The second and third, Waugh et al. [15] and Desclozeaux et al. [16], were included in our current analysis. In our current analysis, we found that koalas vaccinated with MOMP tended to have higher IL17 production six months post-vaccination compared to non-vaccinated koalas in models based on urogenital data (Figure 2d). This was supported by three hypotheses (out of four) based on ocular data (Table 2). This increase can be seen by inspecting the raw data as the average log transformed production of IL17 was higher in vaccinated compared to nonvaccinated groups, $1.616 \pm 0.430(95 \% \mathrm{Cl}, \mathrm{n}=25)$ and $0.462 \pm 0.279(95 \% \mathrm{Cl}, \mathrm{n}=12)$, respectively (Appendix 5). Additionally, we found no clear evidence for a direct relationship between IL17 production and either ocular or urogenital disease that developed six months post-vaccination. Though IL17 is one signalling component of a much larger complex immune response, we found that koalas with a lower chlamydial load produced more IL17 in both the urogenital and ocular sites. 
Both studies by Waugh et al. [15] and Desclozeaux et al. [16] evaluated the effect of MOMP vaccination on subsequent chlamydial disease, using univariate statistical methods. Waugh et al. [15] found that fewer koalas developed disease after 12 months in the MOMP vaccinated group (1 of 23 koalas) compared to control animals (4 of 27 koalas). This result however, was not statistically significant $\left(X^{2}=1.512, p=0.363\right)$ likely owing to the fact that many chlamydial infections remain subclinical and that the total sample size $(n=50)$ may have been too small to determine an effect of vaccination on disease. Desclozeaux et al. [16] found that fewer koalas in the MOMP vaccinated group developed chlamydiosis (either ocular or urogenital chlamydial disease) compared to control koalas ( 0 of 21 and 3 of 21 for MOMP and control koalas, respectively), though this comparison was not evaluated using statistical tests. As well, all MOMP vaccinated koalas reduced their chlamydial load 6-months postvaccination (6 of 21 koalas) and this effect was statistically significant compared to control koalas ( 0 of 21 koalas; $p=0.048)$.

By combining data from both trials, we were able to expand upon these analyses by introducing additional realistic complexity using structural equation models. Preliminary structural equation models indicated that a direct relationship between MOMP vaccination status and subsequent disease development failed our fitting criterion. Given this result, we created models where MOMP vaccination status indirectly affected disease status six months post-vaccination through direct relationships with either chlamydial load or an immune parameter. Multiple models using ocular data and one model using urogenital data (Table 2) indicated that MOMP vaccination status had a direct negative effect on chlamydial load. These effects, however, were lost when we averaged all the urogenital models together. It is possible that given a larger sample size, we could have detected an effect. We performed a power analysis to determine the necessary sample size to detect a univariate effect of MOMP vaccination on urogenital chlamydial load. After estimating an effect size (Cohen's d) to estimate the effect of MOMP vaccination on urogenital chlamydial load [27], we found that a sample size of 117 koalas would be necessary to have an $80 \%$ chance of detecting an effect at a significance level of 0.05 . This finding suggests that an effect might be obtained if a large-scale study included nearly three times the number of koalas that were used in our analysis.

Chlamydial load is among one of several factors that affect disease pathogenesis in free-ranging koalas [26]. Specifically, Quigley et al. [26] found that measurements of chlamydial load (separated into 3 ordinal categories of severity) are strong predictors of urogenital disease, but not ocular disease. In our current study, we also found that koalas with a higher urogenital chlamydial loads six months post-vaccination were more likely to progress to urogenital chlamydial disease when the data were modelled with IL17 production. We did not detect this effect in models of ocular chlamydial load and disease, though this was likely due to a low prevalence of koalas with ocular disease $(n=1)$. Previous studies have suggested that disease pathogenesis in koalas is complex $[17,46]$. A large amount of variation in the prevalence of urogenital disease six months post-vaccination remains unexplained from even our best models $\left(R^{2}=0.268\right)$. Including more factors that are currently associated with disease pathogenesis, including koala retrovirus (KoRV) status, may improve the predictability of these models. 


\section{Conclusions}

In this study, we used structural equation models to identify direct and indirect relationships underpinning vaccination against chlamydial disease in free-ranging koalas. The use of structural equation modelling has previously been applied to identify complex factors influencing disease pathogenesis in koalas [26]. One factor, urogenital chlamydial load, was positively associated with urogenital disease prevalence, agreeing with the findings of Quigley et al. [26]. We found that MOMP vaccination may indirectly affect the development of urogenital disease in koalas by inhibiting the growth of Chlamydia (i.e. lower chlamydial load compared to control koalas). IL17 production is one immune parameter that was increased in MOMP vaccinated koalas. Chlamydial load was a negative predictor of IL17 production, suggesting that the inhibition of chlamydial growth may be reduced by this immune cytokine. Collectively, the structural equation models in this study provide support that IL 17 production is an important immune factor linking vaccination to the reduction of chlamydial load and pathogenesis in free-ranging koalas. More studies using free-ranging animals may be needed to determine the effect of multiple immune parameters on chlamydial load and disease and may make it possible to detect a direct effect of MOMP vaccination on chlamydial load.

\section{Declarations}

\section{Ethics Approval}

Collection of koala data previously collected by Waugh et al. [15] and Desclozeaux et al. [16] were approved by the University of the Sunshine Coast (USC) Animal Ethics Committee (AN/A/13/80) and the Queensland Government (Scientific Purposes Permit, WISP11532912).

\section{Consent for publication}

Not applicable.

\section{Availability of data and materials}

Most of the data generated or analysed during this study are included in the published articles, $[15,16]$. Measurements of cytokine production for IL17 and IFNY in the current study are available from the corresponding author on reasonable request.

\section{Competing interests}

The authors declare that they have no competing interests.

\section{Funding}


This project was supported by the Australian Research Council (Linkage Project 160100138) to P. Timms and S. Carver. Funding for original data collection awarded to P. Timms, A. Polkinghorne, and K. Beagley (LP1202000051) by the Australian Research Council. The overall koala work was supported by many groups including significant support by the Queensland Government (Department of Transport and Main Roads) and specifically the Moreton Bay Rail project team. J. Hanger was supported by Endeavour Veterinary Ecology in the form of a salary.

\section{Authors' contributions}

DL, PT, BLQ, and SC conceived the study design, PT and JH collected the data, DL performed the analysis, and all authors contributed to interpreting the results and drafting of the manuscript.

\section{Acknowledgements}

We thank C.X. Cunningham for his modelling advice and C.L. Exton for her koala illustration. We thank the many groups that supported the overall koala data collection, specifically, C. Waugh et al. (S.A. Khan, J. Loader, and K. Beagley), and M. Desclozeaux et al. (A. Robbins, M. Jelocnik, A. Polkinghorne) for collecting the data published in [15] and [16], respectively, that were used in the current study.

\section{References}

1. Melzer A, Carrick F, Menkhorst P, Lunney D, John BS. Overview, critical assessment, and conservation implications of koala distribution and abundance. Conservation Biology. 2000;14 3:619-28.

2. McAlpine C, Lunney D, Melzer A, Menkhorst P, Phillips S, Phalen D, et al. Conserving koalas: a review of the contrasting regional trends, outlooks and policy challenges. Biological Conservation. 2015;192:226-36.

3. Adams-Hosking C, McBride MF, Baxter G, Burgman M, De Villiers D, Kavanagh R, et al. Use of expert knowledge to elicit population trends for the koala (Phascolarctos cinereus). Diversity and Distributions. 2016;22 3:249-62.

4. Woinarski JC, Burbidge AA, Harrison PL. Ongoing unraveling of a continental fauna: decline and extinction of Australian mammals since European settlement. Proceedings of the National Academy of Sciences. 2015;112 15:4531-40.

5. Polkinghorne A, Hanger J, Timms P. Recent advances in understanding the biology, epidemiology and control of chlamydial infections in koalas. Veterinary microbiology. 2013;165 3-4:214-23.

6. Rhodes JR, Ng CF, de Villiers DL, Preece HJ, McAlpine CA, Possingham HP. Using integrated population modelling to quantify the implications of multiple threatening processes for a rapidly declining population. Biological conservation. 2011;144 3:1081-8.

7. McCallum H, Kerlin DH, Ellis W, Carrick F. Assessing the significance of endemic disease in conservation-koalas, Chlamydia, and koala retrovirus as a case study. Conservation Letters. 
2018;11 4:e12425.

8. Beyer HL, de Villiers D, Loader J, Robbins A, Stigner M, Forbes N, et al. Management of multiple threats achieves meaningful koala conservation outcomes. Journal of applied ecology. 2018;55 4:1966-75.

9. Jackson M, White N, Giffard P, Timms P. Epizootiology of Chlamydia infections in two free-range koala populations. Veterinary microbiology. 1999;65 4:255-64.

10. Jackson M, Giffard P, Timms P. Outer membrane protein A gene sequencing demonstrates the polyphyletic nature of koala Chlamydia pecorum isolates. Systematic and Applied Microbiology. 1997;20 2:187-200.

11. Loader J. An investigation of the health of wild koala populations in South-East Queensland. Bachelor of Applied Science (Animal Studies) Honours thesis, School of Animal Studies, The University of Queensland. 2010.

12. Robbins A, Loader J, Timms P, Hanger J. Optimising the short and long-term clinical outcomes for koalas (Phascolarctos cinereus) during treatment for chlamydial infection and disease. PloS one. 2018;13 12:e0209679.

13. Craig AP, Hanger J, Loader J, Ellis WA, Callaghan J, Dexter C, et al. A 5-year Chlamydia vaccination programme could reverse disease-related koala population decline: Predictions from a mathematical model using field data. Vaccine. 2014;32 33:4163-70.

14. Phillips S, Quigley BL, Timms P. Seventy years of Chlamydia vaccine research-limitations of the past and directions for the future. Frontiers in Microbiology. 2019;10 70.

15. Waugh C, Khan SA, Carver S, Hanger J, Loader J, Polkinghorne A, et al. A prototype recombinantprotein based Chlamydia pecorum vaccine results in reduced chlamydial burden and less clinical disease in free-ranging koalas (Phascolarctos cinereus). PloS one. 2016;11 1:e0146934.

16. Desclozeaux M, Robbins A, Jelocnik M, Khan SA, Hanger J, Gerdts V, et al. Immunization of a wild koala population with a recombinant Chlamydia pecorum Major Outer Membrane Protein (MOMP) or Polymorphic Membrane Protein (PMP) based vaccine: New insights into immune response, protection and clearance. PloS one. 2017;12 6:e0178786.

17. Madden D, Whaite A, Jones E, Belov K, Timms P, Polkinghorne A. Koala immunology and infectious diseases: How much can the koala bear? Developmental \& Comparative Immunology. 2018;82:17785.

18. Lizárraga D, Carver S, Timms P. Navigating to the most promising directions amid complex fields of vaccine development: a chlamydial case study. Expert review of vaccines. 2019;18 12:1323-37; doi: 10.1080/14760584.2019.1698954.

19. Vasilevsky S, Greub G, Nardelli-Haefliger D, Baud D. Genital Chlamydia trachomatis: understanding the roles of innate and adaptive immunity in vaccine research. Clinical microbiology reviews. 2014;27 2:346-70.

20. Igietseme J, Ukwade C, Ouburg S, Omosun Y, Joseph K. Profile of Anti-Chlamydia Immune Responses in Complicated (Infertile) and Non-complicated (Fertile) Genital Infections. J Clin Immunol 
Immunother. 2015;21.

21. Beatty WL, Belanger TA, Desai AA, Morrison RP, Byrne GI. Tryptophan depletion as a mechanism of gamma interferon-mediated chlamydial persistence. Infection and immunity. 1994;62 9:3705-11.

22. Islam MM, Jelocnik M, Huston WM, Timms P, Polkinghorne A. Characterization of the in vitro Chlamydia pecorum response to gamma interferon. Infection and immunity. 2018;86 4:e00714-17.

23. Zhang $Y$, Wang $H$, Ren J, Tang $X$, Jing $Y$, Xing D, et al. IL-17A synergizes with IFN-Y to upregulate iNOS and NO production and inhibit chlamydial growth. PloS one. 2012;7 6:e39214.

24. Refaat B, Al-Azemi M, Geary I, Eley A, Ledger W. Role of activins and inducible nitric oxide in the pathogenesis of ectopic pregnancy in patients with or without Chlamydia trachomatis infection. Clin Vaccine Immunol. 2009;16 10:1493-503.

25. Agrawal T, Bhengraj AR, Vats V, Salhan S, Mittal A. Expression of TLR 2, TLR 4 and iNOS in cervical monocytes of Chlamydia trachomatis-infected women and their role in host immune response. American Journal of Reproductive Immunology. 2011;66 6:534-43.

26. Quigley BL, Carver S, Hanger J, Vidgen ME, Timms P. The relative contribution of causal factors in the transition from infection to clinical chlamydial disease. Scientific reports. 2018;8 1:8893.

27. Borenstein M, Hedges LV, Higgens JP, Rothstein HR. Introduction to Meta-Analysis. Chichester, West Sussex: John Wiley \& Sons, Ltd; 2009.

28. Viechtbauer W. Conducting meta-analyses in R with the metafor package. Journal of statistical software. 2010;36 3.

29. Team RC: R: A language and environment for statistical computing. Vienna, Austria: R Foundation for Statistical Computing; 2017.

30. MacCallum RC, Widaman KF, Zhang S, Hong S. Sample size in factor analysis. Psychological methods. 1999;4 1:84.

31. Kline RB. Principles and practice of structural equation modeling. Guilford publications; 2015.

32. Bollen KA, Stine RA. Bootstrapping goodness-of-fit measures in structural equation models. Sociological Methods \& Research. 1992;21 2:205-29.

33. Kim H, Millsap R. Using the bollen-stine bootstrapping method for evaluating approximate fit indices. Multivariate behavioral research. 2014;49 6:581-96.

34. Anderson D, Burnham K. Model selection and multi-model inference. Second NY: Springer-Verlag. $2004 ; 63$.

35. Burnham KP, Anderson DR, Huyvaert KP. AIC model selection and multimodel inference in behavioral ecology: some background, observations, and comparisons. Behavioral ecology and sociobiology. 2011;65 1:23-35.

36. Rosseel Y. Lavaan: An R package for structural equation modeling and more. Version 0.5-12 (BETA). Journal of statistical software. 2012;48 2:1-36.

37. Dorresteijn I, Schultner J, Nimmo DG, Fischer J, Hanspach J, Kuemmerle T, et al. Incorporating anthropogenic effects into trophic ecology: predator-prey interactions in a human-dominated 
landscape. Proceedings of the Royal Society B: Biological Sciences. 2015;282 1814:20151602.

38. Brunham RC, Rey-Ladino J. Immunology of Chlamydia infection: implications for a Chlamydia trachomatis vaccine. Nature reviews immunology. 2005;5 2:149.

39. Beatty WL, Byrne GI, Morrison RP. Morphologic and antigenic characterization of interferon gammamediated persistent Chlamydia trachomatis infection in vitro. Proceedings of the National Academy of Sciences. 1993;90 9:3998-4002.

40. Cheng C, Pal S, Tifrea D, Jia Z, Luis M. A vaccine formulated with a combination of TLR-2 and TLR-9 adjuvants and the recombinant major outer membrane protein elicits a robust immune response and significant protection against a Chlamydia muridarum challenge. Microbes and infection. 2014;16 3:244-52.

41. Cheng C, Pal S, Bettahi I, Oxford KL, Barry PA, Luis M. Immunogenicity of a vaccine formulated with the Chlamydia trachomatis serovar $\mathrm{F}$, native major outer membrane protein in a nonhuman primate model. Vaccine. 2011;29 18:3456-64.

42. Scurlock AM, Frazer LC, Andrews CW, O'Connell CM, Foote IP, Bailey SL, et al. Interleukin-17 contributes to generation of Th1 immunity and neutrophil recruitment during Chlamydia muridarum genital tract infection but is not required for macrophage influx or normal resolution of infection. Infection and immunity. 2011;79 3:1349-62.

43. Andrew DW, Cochrane M, Schripsema JH, Ramsey KH, Dando SJ, O'Meara CP, et al. The duration of Chlamydia muridarum genital tract infection and associated chronic pathological changes are reduced in IL-17 knockout mice but protection is not increased further by immunization. PloS one. 2013;8 9:e76664.

44. Mathew M, Pavasovic A, Prentis PJ, Beagley KW, Timms P, Polkinghorne A. Molecular characterisation and expression analysis of Interferon gamma in response to natural Chlamydia infection in the koala, Phascolarctos cinereus. Gene. 2013;527 2:570-7.

45. Khan SA, Desclozeaux M, Waugh C, Hanger J, Loader J, Gerdts V, et al. Antibody and cytokine responses of koalas (Phascolarctos cinereus) vaccinated with recombinant chlamydial major outer membrane protein (MOMP) with two different adjuvants. PloS one. 2016;11 5:e0156094.

46. Wan C, Loader J, Hanger J, Beagley K, Timms P, Polkinghorne A. Using quantitative polymerase chain reaction to correlate Chlamydia pecorum infectious load with ocular, urinary and reproductive tract disease in the koala (Phascolarctos cinereus). Australian veterinary journal. 2011;89 10:409-12.

\section{Tables}

Due to technical limitations, tables are only available as a download in the supplemental files section

\section{Figures}


a

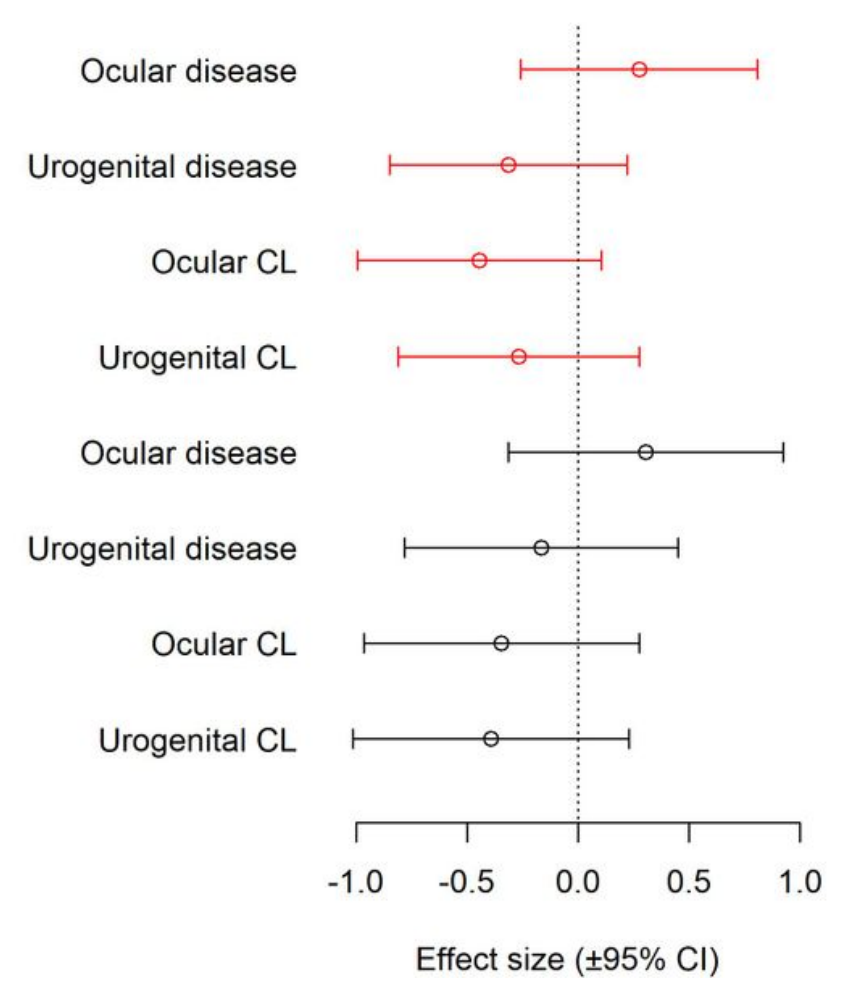

b

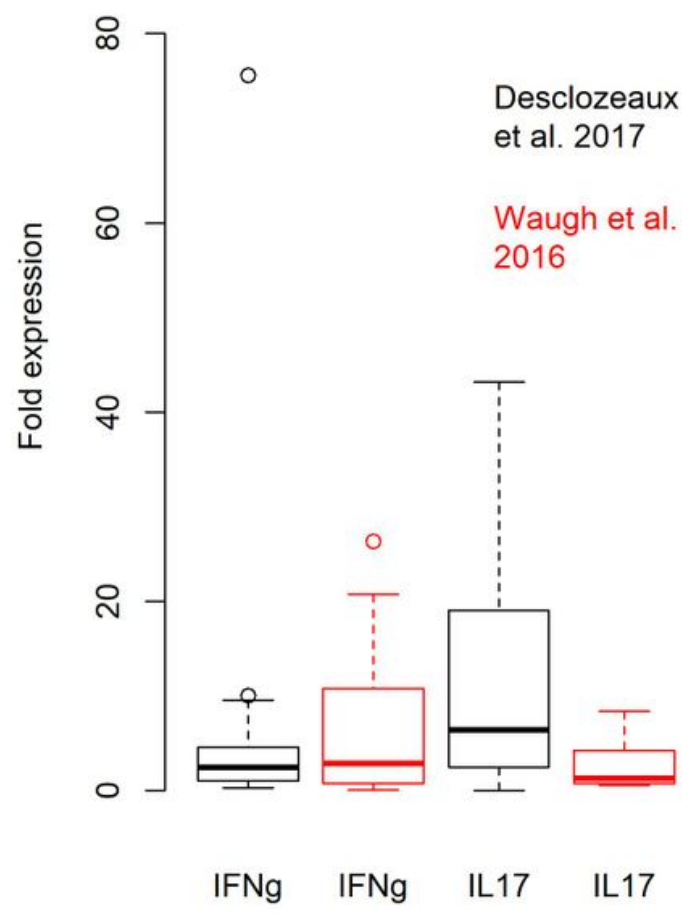

\section{Figure 1}

Comparison of disease and chlamydial load (CL) data collected at the urogenital and ocular sites (a), and interferon y (IFNg) and interleukin 17 expression (IL17; b) collected from wild koalas in two published MOMP-vaccination trials: Desclozeaux et al. 2017 (black effect sizes and boxplots) and Waugh et al. 2016 (red effect sizes and boxplots). Despite differences between the two trials, we found no evidence against pooling data between the two trials after performing a meta-regression comparing the effect of MOMP vaccination on disease and chlamydial load comparing treated and control koalas (metaregression of effect size, $g$ by field trial, $p=0.956$ ). Immune cytokine measurements (fold gene expression relative to the housekeeping gene GADPH) from vaccinated koalas were comparable between the two studies after performing a non-parametric Wilcoxon rank sum test for IFNg or IL17 ( $p=0.9095$, and $p=0.1191$, respectively). 


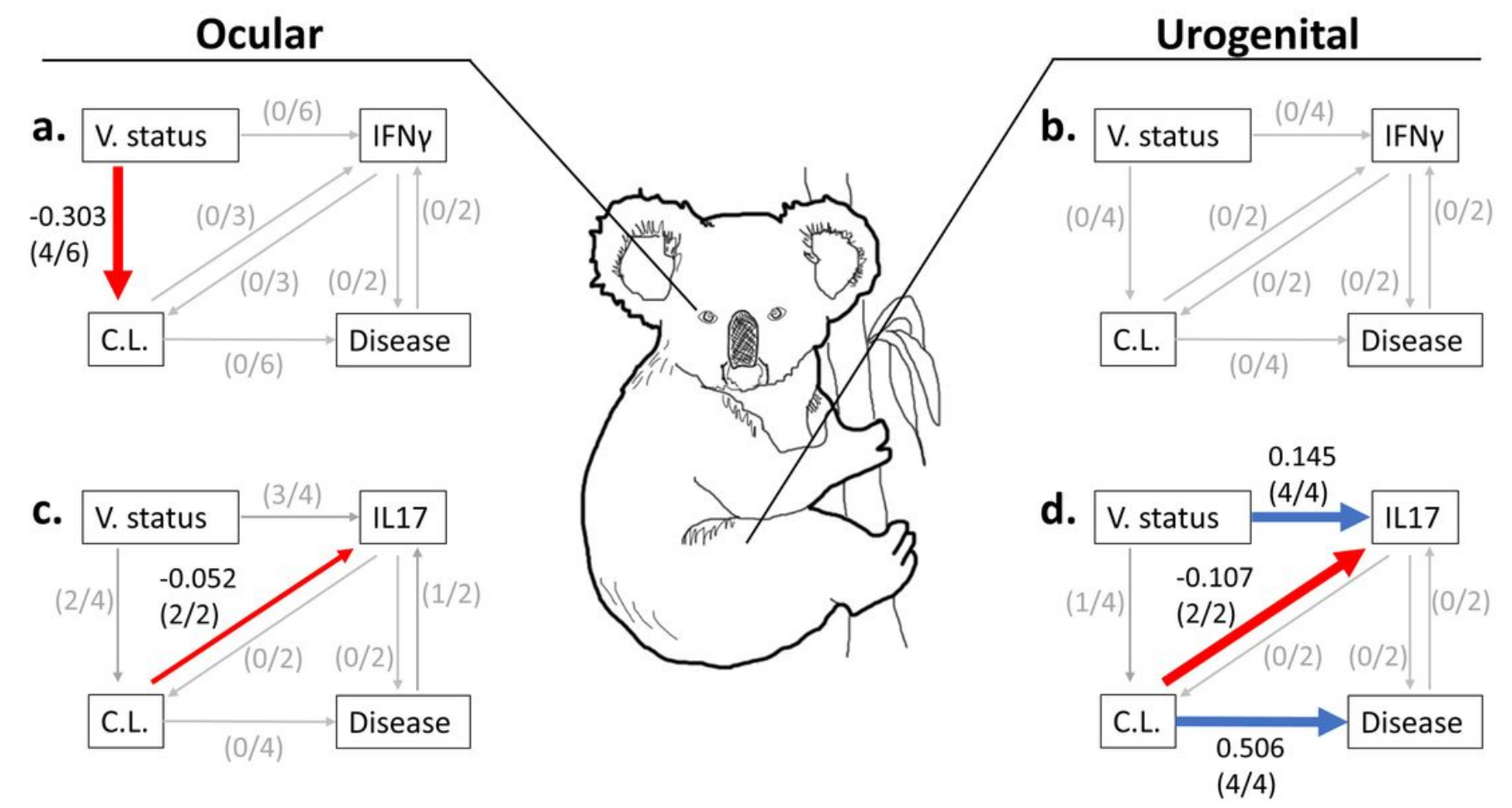

Figure 4

Summary figure of averaged models in Table 4 for ocular (a and c) and urogenital (b and d) data tested with either the expression of IFNy ( $a$ and b) or IL17 ( $c$ and d). Red and blue lines represent relationships with a negative or positive coefficient, respectively, where the coefficient and its variance $(95 \% \mathrm{Cl})$ did not cross 0 (i.e. the threshold of effect). Very small gray lines represent tested relationships in the averaged model where coefficients and its variance $(95 \% \mathrm{Cl})$ crossed 0 . The number of hypotheses with a clear relationship (where the coefficient and its $95 \% \mathrm{Cl}$ did not cross 0 ) between two variables are listed in parentheses. Note: Care should be made in the interpretation of this summary figure as it does not represent the results from any single model, but across all best fitting models using a model averaging approach. The directionality and magnitude of each coefficient shown in this figure are supported by significant coefficients (i.e. coefficients with $95 \%$ Cls that do not cross 0 ) obtained from the averaged models in Table 2. V. status = MOMP vaccination status, C.L. = chlamydial load, Disease $=$ chlamydial disease

\section{Supplementary Files}

This is a list of supplementary files associated with this preprint. Click to download.

- Table114Jan2020.docx

- Graphicalabstractimage14Jan2020.jpg

- AdditionalFile114Jan2020.docx 
- Table114Jan2020.docx

- Graphicalabstractimage14Jan2020.jpg

- AdditionalFile114Jan2020.docx

- Table214Jan2020.docx

- Table214Jan2020.docx 